\title{
A Proposed Nanofabrication Technique Using Optical Masks for Metastable Atom Lithography
}

\author{
M.Baker, A.J. Palmer, and R.T. Sang \\ Centre for Quantum Dynamics, School of Science, \\ Griffith University, Nathan QLD 4111 Australia \\ Email: R.Sang@griffith.edu.au \\ Telephone: +61 73875 3758, Fax: +61 738757656
}

\begin{abstract}
We present an overview of our progress towards using optical fields as 'light masks' for metastable atomic beam lithography. Numerical simulations have been undertaken to model the interaction of a beam of metastable neon atoms with a standing wave of $640.2 \mathrm{~nm}$ laser light. The current experimental setup and the proposed scheme for focusing the atoms is detailed.
\end{abstract}

\section{Keywords-atom lithography; nanofabrication; atom optics}

\section{INTRODUCTION}

In recent years, great advances have been made in the field of atom optics. In particular, the application of optical forces to various atomic species has seen the successful creation of a number of nanofabricated surfaces [1,2]. Atom lithography is a technique that makes use of the manipulation of atomic species using optical forces [3]. Unlike conventional lithography, where various physical masks and lenses are used to manipulate a beam of light, atom lithography employs the light masks to manipulate a beam of atoms. The interest in atom lithography has spawned from the necessity to develop new lithography technologies to overcome the diffraction imposed resolution limits encountered in optical lithography. By using atoms for lithography, having a de Broglie wavelength on the order of picometres, diffraction effects are not a limiting factor. Neutral atoms also have the added advantage of avoiding the space charge effects associated with charged particle techniques such as e-beam and ion beam lithography.

To date, atom lithography has been used in conjunction with a number of atomic species $[2,4,5,6]$. Noble gas atoms in metastable states $\left({ }^{3} \mathrm{P}_{2}\right.$ and ${ }^{3} \mathrm{P}_{0}$ for metastable neon, $\left.\mathrm{Ne}^{*}\right)$ possess sufficient internal energy $(\sim 16 \mathrm{eV})$ to prove useful to the lithography process. In our work, we have investigated the use of alkanethiol self assembled monolayers $\left(\mathrm{C}_{n} \mathrm{H}_{2 n+1} \mathrm{SH}\right)$ [4]. Upon collision with the surface, the energy of the metastable atom is used to break or form carbon bonds in the resist layer. With appropriate etching techniques, the pattern can be transferred to the underlying substrate.

In previous work, we have used our metastable Ne* source to pattern $\mathrm{Au} / \mathrm{Si}$ substrates coated with alkanethiol resists using mechanical proximity masks [9]. Our apparatus has now been expanded to investigate optical masking techniques. We present here our recent work to investigate the use of optical standing waves as masks to produce periodic patterns on the nanometer scale. These masks make use of the optical dipole force, resulting from the interaction of the induced atomic dipole with the gradient in the optical field intensity $[3,7]$.

As we will show, the 1D standing wave acts as a set of cylindrical lenses for the atoms. Numerical simulations have been performed for $\mathrm{Ne}^{*}$ which show periodic structures with features $<100 \mathrm{~nm}$ are easily attainable with modest system requirements. An overview of the experimental apparatus is presented, detailing the operation of the source, the atomic beam collimating section, and the proposed scheme to realize the standing wave focusing.

\section{SEMI-CLASSICAL MODEL OF THE DIPOLE FORCE ON NE* ATOMS IN A STANDING WAVE}

Consider an atom with longitudinal velocity in the $z$ direction $\left(v_{z}\right)$, and transverse components in the $\mathrm{x}$ and $\mathrm{y}$ directions $\left(v_{x}, v_{y}\right.$ respectively), passing through a light field.

The potential for the dipole force on a two-level atom in a laser field can be described by $[7,8]$

$$
U(x, y, z)=\frac{\hbar \gamma^{2}}{8 \Delta} \frac{I(x, y, z)}{I_{s}}
$$

Here $\gamma$ is the linewidth of the atomic transition, $\Delta$ the detuning of the laser to the transition, $I(x, y, z)$ is the intensity of the laser field at a particular point along the beam, and $I_{s}$ is the saturation intensity. The potential detailed in (1) is valid assuming two conditions; that the atom moves slowly enough through the laser beam for its internal state to be in equilibrium with the radiation field (i.e. adiabatically), and that it does not spend long enough in the laser beam for spontaneous emission events to impart kinetic energy to the atom and alter its trajectory. Under these conditions, the potential can be written as $[7,8]$

$$
U(x, y, z)=\frac{\hbar \Delta}{2} \ln \left[1+\frac{I(x, y, z)}{I_{s}} \frac{\gamma^{2}}{\gamma^{2}+4 \Delta^{2}}\right]
$$


For a standing wave along the $x$ direction, with a Gaussian beam profile ( $y$ and $z$ direction), the intensity can be described by

$$
I(x, y, z)=I_{0} \exp \left(-\left(\frac{2 y^{2}}{w_{y}^{2}}+\frac{2 z^{2}}{w_{z}^{2}}\right)\right) \sin ^{2} k x
$$

Here $w_{y}$ and $w_{z}$ are the $1 / \mathrm{e}^{2}$ widths of the beam in the $y$ and $z$ directions, and $I_{0}$ is the intensity of the beam at the antinodes of the standing wave. The dipole force experienced by the atoms is then simply given by

$$
F(x, y, z)=-\vec{\nabla} U(x, y, z)
$$

Each node of the standing wave acts as a lens, and the entire standing wave behaves as a periodic set of cylindrical lenses for the atoms. The power required for focusing the atoms to the centre of the Gaussian beam is given by

$$
P_{f}=5.37 \frac{\pi E_{0} I_{s} \Delta}{\hbar \gamma^{2} k^{2}}
$$

and the laser intensity $I_{0}$ is related to the traveling power by

$$
I_{0}=\frac{8 P_{f}}{\pi w_{z}^{2}}
$$

We have solved the equations of motion (4) using a beam of $\mathrm{Ne}^{*}$ in the ${ }^{3} \mathrm{P}_{2}$ state traversing a standing wave of laser light detuned from the ${ }^{3} \mathrm{P}_{2}-{ }^{3} \mathrm{D}_{3}$ transition, and monitored the atomic trajectories. The parameters chosen for the $\mathrm{Ne}^{*}$ beam are consistent with our system, where $v_{z}=540 \mathrm{~m} / \mathrm{s}, \gamma=5.32 \times 10^{7}$ $\mathrm{rad} \mathrm{s}^{-1}, \lambda=640.2 \mathrm{~nm}$, with a detuning of $\Delta=2 \pi \times 150 \mathrm{MHz}$ and a beam width of $w_{y}, w_{z}=300 \mu \mathrm{m}$. The saturation intensity for the transition is $42 \mathrm{~W} \mathrm{~m}^{-2}$. The power required for focusing under these conditions is then calculated to be $P_{f}=0.11 \mathrm{~mW}$.

Figure 1 shows a summary of typical results of the trajectories using these parameters but with different velocity conditions of the atomic beam. The plots represent the flux distributions of atoms in the focal plane of the substrate. In Figure 1(a), the atomic beam was calculated with a longitudinal velocity spread, calculated from time-of-flight and beam temperature measurements [11], and beam spread of $5.4 \times 10^{-4} \mathrm{rad}$ (FWHM), corresponding to transverse velocities of $v_{x}, v_{y} \sim 25 \mathrm{~cm} / \mathrm{s}$. Figure 1(b) shows the case of no longitudinal velocity spread, but with a transverse velocity spread. Conversely, Figure 1(c) shows the situation with no transverse velocities, but with a longitudinal velocity spread.

Under these conditions, we can see that structures of $72 \mathrm{~nm}$ are predicted for the realistic thermal beam accounting for transverse and longitudinal velocities (see Figure 1(a)). The effect of varying longitudinal and transverse velocities is analogous to chromatic and spherical aberration respectively in conventional lens optics. Comparison of the atomic flux distributions for the three cases presented in Figure 1 shows (a)

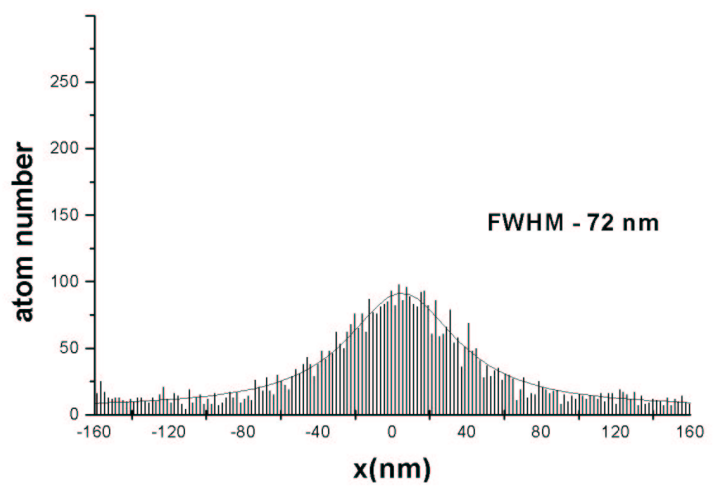

(b)

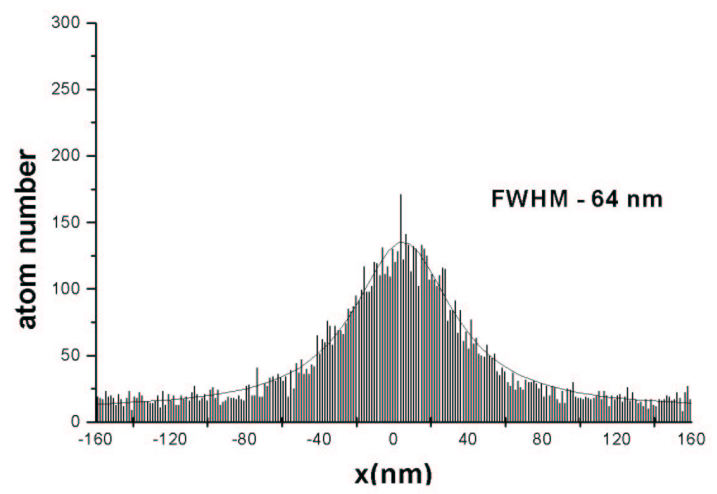

(c)

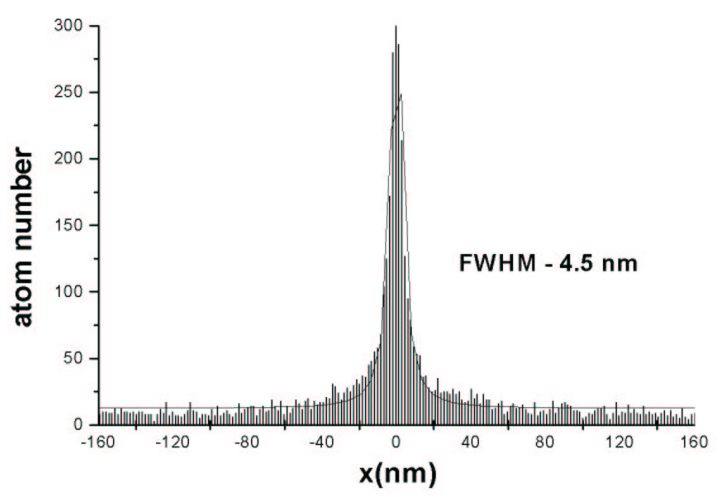

Figure 1. Comparison of atomic flux distributions for $\mathrm{Ne}^{*}$ (a) The atoms have a thermal longitudinal velocity spread, and angular spread of $5.4 \times 10^{-4}$ rad (FWHM) (b) Atoms have no longitudinal spread, but an angular apread of of $5.4 \times 10^{-4}$ (FWHM) (c) Atoms have a thermal longitudinal velocity spread, but zero transverse velocities.

that the major limiting factor in the resolution obtained is in the spread of transverse velocities. The effect of the longitudinal spread in velocities is less critical. One can see that for the standing wave focusing to be effective, a well-collimated beam with low transverse velocities is required. 


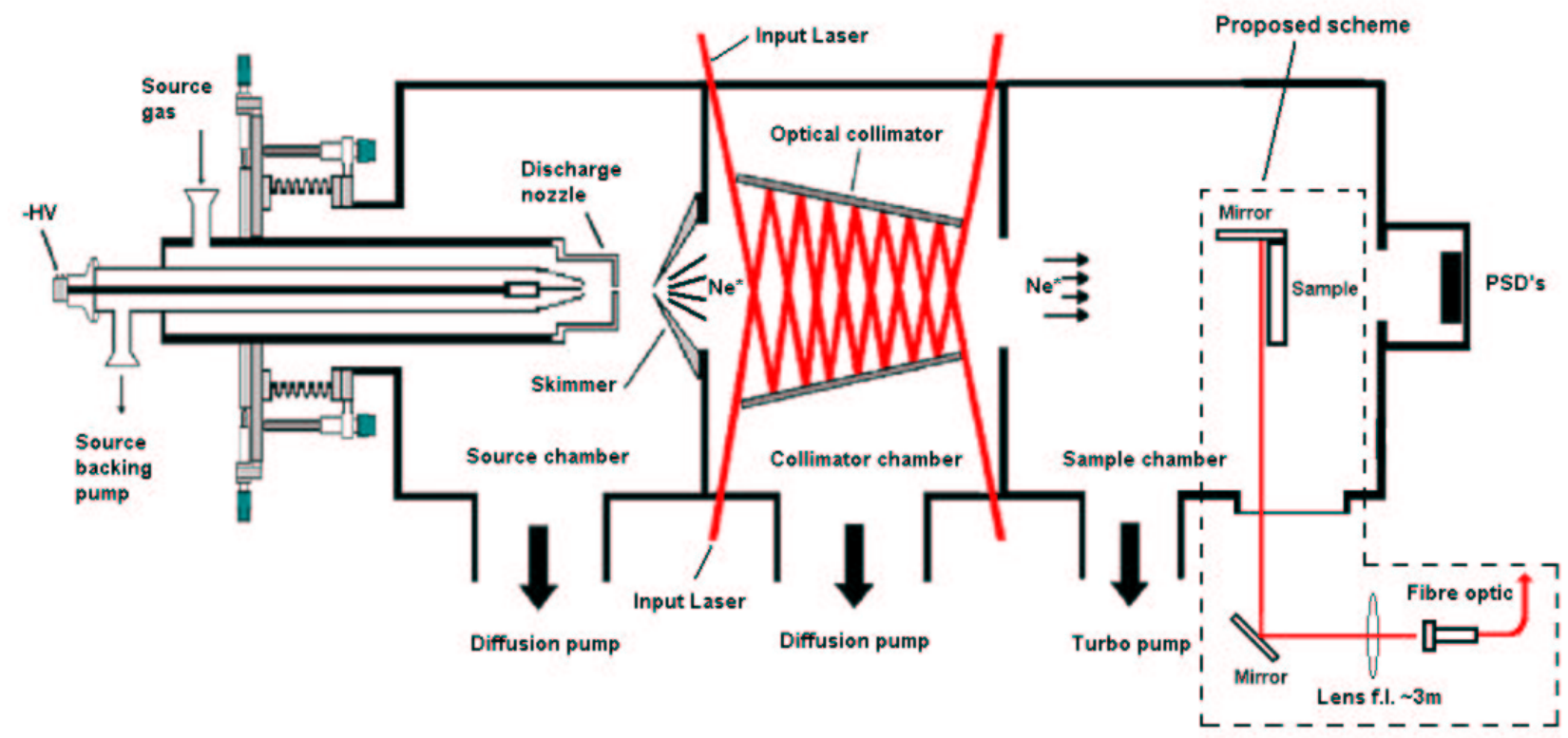

Figure 2. Atom lithography apparatus, showing $\mathrm{Ne}^{*}$ source, collimating section, and proposed scheme for standing wave focusing of the atomic beam

\section{EXPERIMENTAL SETUP}

The apparatus consists of three principal sections: the metastable neon source, the multi-reflection atomic beam collimator, and the sample chamber (see Figure 2). All elements of the system are mounted on a vibration stable optical table. The metastable atomic beam is produced using a liquid nitrogen cooled DC discharge source. Neon gas expands supersonically from a $300 \mu \mathrm{m}$ boron nitride nozzle. As the gas expands, a discharge is created between a cathode and a skimmer plate with a $1.5 \mathrm{~mm}$ aperture. Electron collisions with the $\mathrm{Ne}$ excite a proportion $(\sim 0.01 \%)$ of the atoms to the metastable ${ }^{3} \mathrm{P}_{2}$ and ${ }^{3} \mathrm{P}_{0}$ states, with an internal energy of $\sim 16$ $\mathrm{eV}$. Atoms not excited remain in the inert ground state. Any ions and free electrons produced in the discharge are deflected from the beam path by a magnetic field. During operation, the source generates a Ne* beam brightness of $3.8 \times 10^{14}$ atoms sr $\mathrm{s}^{-1}$, with a most probable longitudinal velocity of $540 \mathrm{~m} / \mathrm{s}$.

Upon passing through the skimmer aperture, the metastable atoms undergo transverse laser cooling in the collimation section. The collimator makes use of the spontaneousemission force resulting from the absorption and the random emission of photons by the $\mathrm{Ne}^{*}$ atoms. The collimator consists of an ensemble of two sets of two slightly off parallel mirrors (one set for each of the transverse directions, $x$ and $y$ ). Four laser beams of $640.2 \mathrm{~nm}$ wavelength, corresponding to the ${ }^{3} \mathrm{P}_{2}$ $-{ }^{3} \mathrm{D}_{3}$ closed transition in $\mathrm{Ne}^{*}$, undergo 40 reflections down the lengths of the mirrors. As the light reflects down the mirrors, the angle of incidence with the atomic beam changes throughout the interaction region. This ensures that the light field that the atoms encounter in the interaction region has an effective detuning corresponding to the Doppler shifted frequency required for the atoms to be in resonance with the transition. Under these conditions, the atoms are continually cooled in the transverse direction through the entire collimator.

The initial detuning of the input beams is chosen to be $\sim 60$ $\mathrm{MHz}$, corresponding to atoms with a transverse velocity $v_{x}, v_{y} \sim$ $50 \mathrm{~ms}^{-1}$. The length of the collimator mirrors is $16 \mathrm{~cm}$, the initial input angle is $120 \mathrm{mrad}$, and the mirrors are set at an angle of $2 \mathrm{mrad}$ from parallel. The laser used is a Spectra Physics dye laser (380D) using DCM dye, pumped by a Coherent $\mathrm{Ar}^{+}$laser (Innova 400). The light is coupled into an optical fiber and transferred to the optical table, where the beam is expanded to $20 \mathrm{~mm}$ diameter, and split into the four collimating beams. The laser provides $21 \mathrm{~mW}$ of power for each collimating beam.

To measure the degree of collimation, a 'cold wire' detector was used, consisting of a $1.3 \mathrm{~mm}$ diameter stainless steel wire mounted on a linear drive able to be positioned into the beam. The highly energetic $\mathrm{Ne}^{*}$ atoms striking the surface of the wire can be detected by Auger emission of an electron from the metal surface, producing a measurable current proportional to the number of $\mathrm{Ne}^{*}$ atoms striking the wire. Figure 3 shows a comparative plot of the increase in atomic beam flux provided by the atomic beam collimator as measured in this way, located $\sim 50 \mathrm{~cm}$ from the collimating section. As can be seen, the collimation section provides a significant increase in atomic flux and beam density, with the peak beam intensity increasing threefold. 


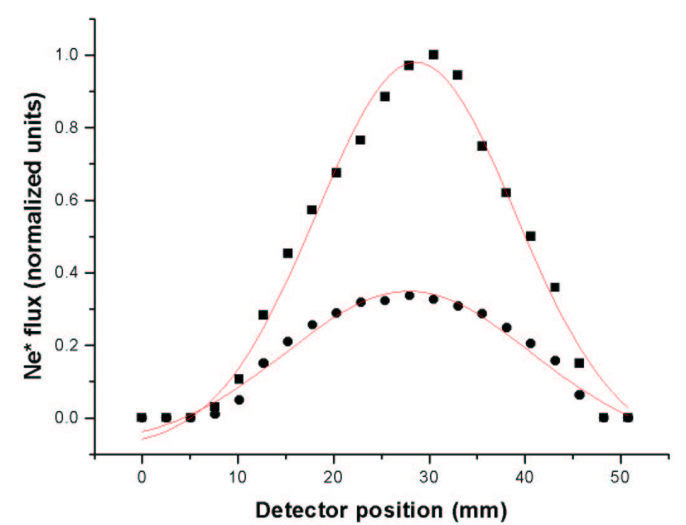

Figure 3. Atomic beam flux increase due to transverse laser cooling. The larger curve shows the beam profile when undergoing transverse laser collimation , the smaller curve with no collimation. The optimum collimation condition is $\sim 60 \mathrm{MHz}$ red detuned from the atomic transition.

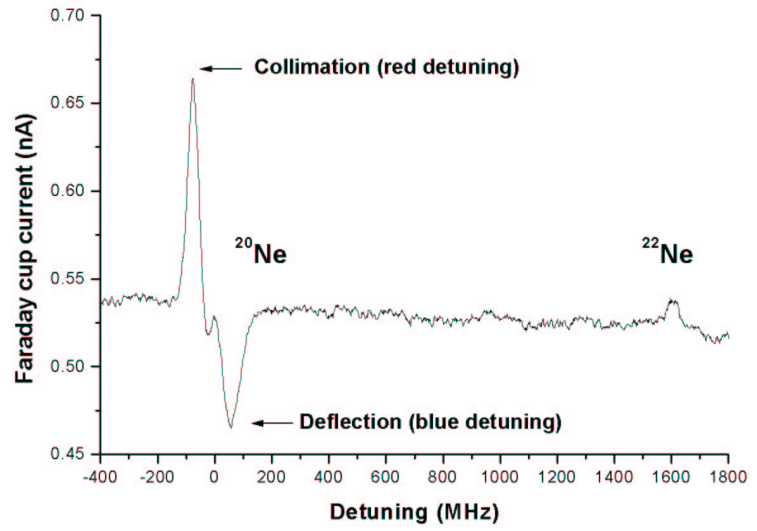

Figure 4. Atomic flux as a function of initial collimation beam detuning. The presence of the secondary peak belongs to the ${ }^{22} \mathrm{Ne}$ isotope.

The increase in atomic flux from the collimator was also measured using a biased stainless steel Faraday cup located 15 $\mathrm{cm}$ from the collimator. Figure 4 shows a plot of the increase in flux as the laser frequency stepped from red to blue detuning. From the plot we can see clearly the optimum initial detuning for the collimator, as well as a corresponding blue detuning condition where atoms are heated and deflected from the collimator. After traveling through the collimating section, the atomic beam, now with reduced transverse velocities and beam divergence, enters the sample chamber. Our samples consist of $\mathrm{Si}<100>$ coated with a thin layer of $\mathrm{Cr}(3 \mathrm{~nm})$ and $\mathrm{Au}(45 \mathrm{~nm})$. To form the resist, the samples are then placed into a $1 \mathrm{mM}$ solution of dodecanethiol (DDT) in ethanol. The samples are then mounted into the chamber, and exposed to the beam. For our $\mathrm{Ne}^{*}$ atomic beam, exposure times of 35 minutes have been adequate to sufficiently damage the resist layer to produce positive patterning [9]. An exposure of this duration corresponds to a dosage of $\sim 9 \times 10^{14}$ atoms $\mathrm{cm}^{-2}\left(\sim 2 \mathrm{Ne}^{*}\right.$ atoms per DDT molecule). Etching of the samples is achieved using a standard ferrocyanide etching solution [4].

\section{PROPOSED SCHEME FOR STANDING WAVE FOCUSING OF THE NE* BEAM}

Also detailed in Figure 2 is our proposed scheme to achieve the standing wave focusing of the atomic beam. In this scheme, laser light from a second laser ring dye laser (Coherent 899) also using DCM dye at the $640.2 \mathrm{~nm}$ transition, is focused into an optical fiber and transferred to the experiment optical table. Frequency locking is made relative to a saturated absorption cell (SAC). Powers up to $100 \mathrm{~mW}$ are produced from the fiber, and the laser allows a detuning of up to $200 \mathrm{MHz}$ without losing the frequency lock condition. The beam is then focused to a Gaussian width of $260 \mu \mathrm{m}$ across the edge of the sample using lenses with a combined focal length of $\sim 3 \mathrm{~m}$. The standing wave is formed by retro-reflecting this beam from a mirror mounted $90^{\circ}$ to the sample. Micrometer adjustments of the sample holder allow the beam to be precisely aligned across the sample, and back along the path length of the beam.

\section{CONCLUSIONS AND FUTURE WORK}

This scheme is currently being implemented, with most of the optical components now in place. To avoid phase shifts in the standing wave caused by seismic and vibration noise, the whole experiment has been mounted on an air-damped optical table. This should avoid the necessity of active feedback to stabilize the detuned standing wave over the sample. We intend to investigate the focusing of the atoms at laser powers existing in the focusing regime $\left(P_{f} \sim 1 \mathrm{~mW}\right)$ and at higher laser powers $\left(P_{f}\right.$ up to $100 \mathrm{~mW}$ ), at which the model described here breaks down, and the atoms undergo channeling in the standing wave. At these high powers, however, the alignment of sample relative to the beam is less critical [10]. Models undertaken by other groups indicate that tighter focusing should also be possible in the high power regime [8]. It is our intention to make a comparison of these two regimes, and extend the standing wave patterning to $2 \mathrm{D}$.

\section{REFERENCES}

[1] J.H. Thywissen, et al, J. Vac. Sci. Technol. B 15 (6), 2093 (1997)

[2] D.Meschede, H.Metcalf, J. Phys. D: Appl. Phys. 36, R17 (2003)

[3] G.Timp, R.E. Behringer, D.M. Tennant, J.E. Cunningham, Phys. Rev. Lett. 69 (11), 1636 (1992)

[4] K.K. Berggren, A. Bard et al, Science 2691255 (1995)

[5] S. Nowak, T. Pfau, J. Mlynek, Appl. Phys. B 63, 203 (1996)

[6] S.B. Hill, C.A. Haich et al, Appl. Phys. Lett. 74 (15), 2239 (1999)

[7] J.J. McClelland, J. Opt. Soc. Am. B, 12 (10), 1761 (1995)

[8] S.J.H. Petra et al, Eur. Phys. J. D 27 (1), 83 (2003)

[9] M.Baker, A.J. Palmer, R.T. Sang, Nanotechnology, 15, 1356 (2004)

[10] K.A.H. van Leeuwen, private communications

[11] A.J. Palmer., M.Baker, R.T. Sang, Rev. Sci. Instrum., 75 (11), 5056 (2004) 